\title{
https://doi.org/10.46813/2021-131-084 \\ RADIATION OF CHARGE PASSING BY IMPEDANCE WEDGE
}

\section{Ostroushko}

\section{National Science Center “Kharkov Institute of Physics and Technology”, Kharkiv, Ukraine E-mail: ostroushko-v@kipt.kharkov.ua}

The diffraction radiation generated by a charge passing by a plasma wedge is considered in impedance approximation. In the perfect conductivity limit, the total radiated energy is not varying with the wedge rotation around the edge fixed along with the charge motion line. The impedance increase may lead to the total radiated energy increase, due to effective surface wave generation, when the charge motion to the edge is almost parallel to a face of wedge.

PACS: 41.60.-m

\section{INTRODUCTION}

A diffraction radiation may be emitted by a bunch of the charged particles passing by a plasma structure, which plays a role of antenna [1]. For effective wideband emission, antenna should have no characteristic length. At the low frequency the antenna may be considered as perfectly conductive. Resistance can be taken into account in impedance approximation. The case when antenna is a perfectly conductive halfplane and the particle motion direction is perpendicular to its edge is studied in [2]. In [3] the direction is arbitrary. In the present work, antenna is a perfectly conductive or an impedance wedge and the charged particle moves uniformly along a line, which does not cross the wedge faces.

\section{PROBLEM FORMULATION}

In the following study, the Cartesian coordinates $(x, y, z), \quad(\xi, \eta, \zeta)$, and $\left(x^{\prime}, y^{\prime}, z\right)$ and the polar coordinates $(r, \theta, \varphi)$ and $(\rho, \varphi, z)$ are used (Fig. 1).

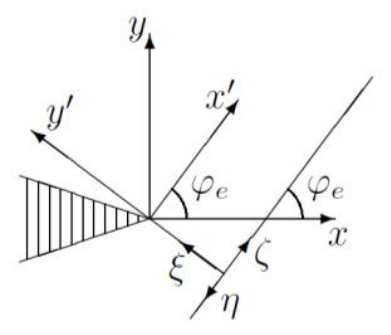

$a$

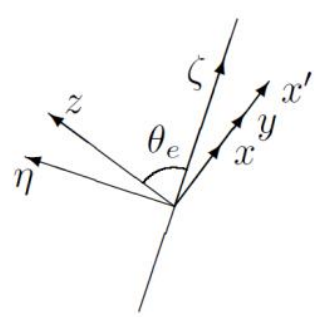

$b$
Fig. 1. The views from $z=+\infty(a)$ and $\xi=-\infty(b)$

The axis $z$ is the edge of wedge, the sector $\varphi \in(-\Phi,+\Phi)$ with $\Phi \in(\pi / 2, \pi)$ is free space, the axis $\zeta$ is the line of the particle's motion, the axis $\xi$ is directed to the edge along the shortest line segment between two points, at the motion line and at the edge, $\xi_{\mathrm{e}}$ denotes the segment length, the axis $x^{\prime}$ is parallel to the motion line projection on a plane perpendicular to the edge, $\varphi_{\mathrm{e}}$ denotes the angle between the directions $x^{\prime}$ and $x, \theta_{\mathrm{e}}$ denotes the angle between the directions $\zeta$ and $z$. It is assumed that the particle moves from the half-space $y<0$ to the half-space $y>0$, and so, the wedge is in the half-space $y^{\prime} \geq 0$, and $\varphi_{\mathrm{e}} \in(\pi-\Phi, \Phi)$.

The coordinates are connected by the equalities $z=\zeta \cos \theta_{\mathrm{e}}+\eta \sin \theta_{\mathrm{e}}$ $x^{\prime}=\zeta \sin \theta_{\mathrm{e}}-\eta \cos \theta_{\mathrm{e}}$, $y^{\prime}=\xi-\xi_{\mathrm{e}}, \quad z=r \cos \theta, \quad \rho=r \sin \theta, \quad x=\rho \cos \varphi$, $y=\rho \sin \varphi, x^{\prime}=\rho \cos \left(\varphi-\varphi_{\mathrm{e}}\right), y^{\prime}=\rho \sin \left(\varphi-\varphi_{\mathrm{e}}\right)$.

The particle motion is determined by the equalities $\xi=0, \eta=0, \zeta=\beta c t$, where $t$ is time, $c$ is the speed of light, $\beta \in(0,1)$. In free space, the electromagnetic field obeys Maxwell's equations, and it may be given by the sum of $\mathrm{E}$ - and $\mathrm{H}$ - waves. The space-time dependence of electric and magnetic field strength components of these waves has the form $\operatorname{Re}\left[\vec{F}(x, y) \exp \left(i k_{z} z-i \omega t\right)\right]$, where $F$ stands for $E$ or $H$. The amplitudes of the longitudinal components obey the equations $\nabla_{\perp}^{2} F_{z}+k_{\perp}^{2} F_{z}=0$, where $\nabla_{\perp}=\vec{e}_{x} \partial_{x}+\vec{e}_{y} \partial_{y}, \quad k_{\perp}=\left(k^{2}-k_{z}^{2}\right)^{1 / 2}, k=\omega / c, \vec{e}$ with indexes are unit vectors of relevant directions, $\partial$ with indexes are derivatives with respect to relevant variables. The amplitudes of the transverse components are determined by ones of the longitudinal components,

$$
\begin{aligned}
& k_{\perp}^{2} \vec{E}_{\perp}=+i k\left[\nabla H_{z} \vec{e}_{z}\right]+i k_{z} \nabla_{\perp} E_{z}, \\
& k_{\perp}^{2} \vec{H}_{\perp}=-i k\left[\nabla E_{z} \vec{e}_{z}\right]+i k_{z} \nabla_{\perp} H_{z} .
\end{aligned}
$$

It is assumed that impedance $\mathrm{Z}$ for both faces is identical and depends on frequency, $\mathrm{Z}=\left(-i k \delta_{\mathrm{s}}\right)^{p_{\mathrm{s}}}$, where $\delta_{\mathrm{s}}$ is skin layer depth and the power index, $p_{\mathrm{s}}$ is connected with the skin effect type. So, $\mathrm{Z}=\left(k \delta_{\mathrm{s}}\right)^{p_{\mathrm{s}}} \exp \left(-i \alpha_{\mathrm{s}}\right)$, where $\alpha_{\mathrm{s}}=p_{\mathrm{s}} \pi / 2$. If one proceeds from the equality $\mathrm{Z}=\left\{1-\omega_{\mathrm{e}}^{2}[\omega(\omega+i v)]^{-1}\right\}^{-1 / 2}$, where $\omega_{\mathrm{e}}$ and $v$ are plasma and collision frequencies then the cases of normal and anomalous skin effect (related to the frequency ranges $\omega<<v<<\omega_{\mathrm{e}}$ and $\left.v<<\omega<<\omega_{\mathrm{e}}\right)$ correspond to $p_{\mathrm{s}}=1 / 2, \quad \delta_{\mathrm{s}}=c v / \omega_{\mathrm{e}}^{2}$, and $p_{\mathrm{s}}=1, \delta_{\mathrm{s}}=c / \omega_{\mathrm{e}}$, respectively, and dependence of $\mathrm{Z}$ on $k$ is near to power one at $k \delta_{\mathrm{s}}<<1$ (and then $|Z|<<1)$.

Both for E-waves, and for $\mathrm{H}$-waves the problem is reduced to the plane one: for the given field of an external source $F_{z}^{\mathrm{e}}$, it should be found the scattered field $F_{z}^{\mathrm{s}}$, which obeys the equation $\nabla_{\perp}^{2} F_{z}^{\mathrm{s}}+k_{\perp}^{2} F_{z}^{\mathrm{s}}=0$, the radiation condition, and Meixner's edge condition, and the full field, $\vec{F}^{\mathrm{f}}=\vec{F}^{\mathrm{s}}+\vec{F}^{\mathrm{e}}$, should obey the impedance boundary conditions, $\pm E_{z}^{\mathrm{f}} / H_{\rho}^{\mathrm{f}}=\mathrm{Z}=\mp E_{\rho}^{\mathrm{f}} / H_{z}^{\mathrm{f}}($ at $\varphi= \pm \Phi)$, which may be 
written in the form $\left(\rho F_{z}^{\mathrm{f}}\right)^{-1} \partial_{\varphi} F_{z}^{\mathrm{f}}= \pm i k_{\perp} \sin \chi_{F}$, where $\chi_{E, H}$ are determined by the equations,

$$
\sin \chi_{E}=k_{\perp} /(k Z), \sin \chi_{H}=\left(k_{\perp} Z\right) / k,
$$

and the condition $0<\operatorname{Re} \chi_{E, H} \leq \pi / 2$.

\section{PROBLEM SOLUTION}

The solution of the problem of one plane wave scattering on impedance wedge is described circumstantially in the original papers and books $[4,5]$. The result is briefly described below. Let the incident scalar plane wave with unit amplitude in the 2D space has the spatial dependence $\exp \left(i k_{x}^{\prime} x^{\prime}+i k_{y}^{\prime} y^{\prime}\right)$, where $k_{x}^{\prime}$ is real and $k_{y}^{\prime}=\left(k_{\perp}^{2}-k_{x}^{\prime 2}\right)^{1 / 2}$. Then the sum of the wave and the scattered field may be given in the form of Sommerfeld integral,

$$
\begin{gathered}
f_{z}(\chi, \rho, \varphi)=(2 \pi i)^{-1} \times \\
\times \int_{C} d w \exp \left(-i k_{\perp} \rho \cos w\right) s_{z}(\chi, \varphi+w) .
\end{gathered}
$$

Here $s_{z}(\chi, w)=s_{0}(w) P_{\Phi}(\chi, w) / P_{\Phi}\left(\chi, w_{\mathrm{i}}\right)$,

$$
\begin{gathered}
s_{0}(w)=k_{\Phi} \cos \left(k_{\Phi} w_{\mathrm{i}}\right) /\left[\sin \left(k_{\Phi} w\right)-\sin \left(k_{\Phi} w_{\mathrm{i}}\right)\right], \\
k_{\Phi}=\pi /(2 \Phi), P_{\Phi}(\chi, w)=\prod_{\{ \pm\}} \psi_{\Phi / 2}((\pi / 2)-\chi \pm w), \\
\psi_{\Phi}(w)=\exp \left\{-\int_{0}^{\infty} d \tau[\operatorname{ch}(\tau w)-1] \times\right. \\
\left.\times[2 \tau \operatorname{ch}(\pi \tau / 2) \operatorname{sh}(2 \Phi \tau)]^{-1}\right\} \\
w_{\mathrm{i}}=\varphi_{\mathrm{e}}-\pi+i \operatorname{arcosh}\left(k_{x}^{\prime} / k_{\perp}\right) .
\end{gathered}
$$

The integration path $C$ in (2) consists of two parts: $C_{+}$, in the half-plane $\operatorname{Im} w>0$, and $C_{-}$, symmetrical to $C_{+}$ with respect to the point $w=0$. The path $C_{+}$goes along the parts of three straight lines, from $\varepsilon^{\prime}+i \infty$ to $-\pi-\varepsilon^{\prime}+i \infty$, passing through the points $\varepsilon^{\prime}+i \varepsilon^{\prime \prime}$ and $-\pi-\varepsilon^{\prime}+i \varepsilon^{\prime \prime}$, consecutively, where $\varepsilon^{\prime}$ and $\varepsilon^{\prime \prime}$ should obey the inequalities $0<\varepsilon^{\prime}<\pi / 2$ and $\varepsilon^{\prime \prime}>\left|\operatorname{Im} w_{\mathrm{i}}\right|$. The quantity $\chi$ should be taken from (1). The quantity $\operatorname{Re} w_{\mathrm{i}}$ corresponds to the angle (with respect to $x^{\prime}$ axis) of the wave incidence direction. The function $s_{0}(w)$ has the pole at $w=w_{\mathrm{i}}$, corresponding to the incident wave. Also, it can have the poles at $w=2 \Phi-w_{\mathrm{i}}$ (if $\left.\operatorname{Re} w_{\mathrm{i}} \in(\Phi-\pi, \Phi)\right) \quad$ and at $\quad w=-2 \Phi-w_{\mathrm{i}} \quad$ (if $\left.\operatorname{Re} w_{\mathrm{i}} \in(-\Phi, \pi-\Phi)\right)$, corresponding to the waves reflected from top and bottom faces.

To use the written relations for the considered problem solving, one has to present the field connected with the moving charge $e_{0}$ by integral Fourier over the plane waves coming to the line $y^{\prime}=0$ in $2 \mathrm{D}$ problem. The time-dependent Liénard-Wiechert potentials are: $\vec{A}_{t}^{\mathrm{e}}=\beta \varphi_{t}^{\mathrm{e}} \vec{e}_{\zeta}, \quad \varphi_{t}^{\mathrm{e}}=e_{0}\left[(\zeta-\beta c t)^{2}+\left(1-\beta^{2}\right)\left(\xi^{2}+\eta^{2}\right)\right]^{-1 / 2}$. For $\{k>0, \xi>0\}$, performing the Fourier transform, $\hat{\varphi}^{\mathrm{e}}=(2 \pi)^{-1} \int_{-\infty}^{+\infty} d t \exp (i k c t) \varphi_{t}^{\mathrm{e}}$, one gets

$$
\begin{aligned}
& \hat{\varphi}^{\mathrm{e}}=e_{0}(\pi \beta c)^{-1} \mathrm{~K}_{0}\left(f_{\perp} \sigma\right) \exp \left(i k \beta^{-1} \zeta\right)= \\
& =\int_{-\infty}^{+\infty} d k_{\eta} \exp \left(i k_{\eta} \eta+i k \beta^{-1} \zeta-\kappa_{\xi} \xi\right) \hat{\varphi}^{\mathrm{e}},
\end{aligned}
$$

where $\sigma=\left(\xi^{2}+\eta^{2}\right)^{1 / 2}, \quad f_{\perp}=k /(\beta \gamma), \quad \gamma=\left(1-\beta^{2}\right)^{-1 / 2}$, $\kappa_{\xi}=\left(f_{\perp}^{2}+k_{\eta}^{2}\right)^{1 / 2}, \quad \hat{\varphi}^{\mathrm{e}}=e_{0}\left(2 \pi \beta c \kappa_{\xi}\right)^{-1}, \quad \mathrm{~K}_{0} \quad$ is
McDonald's function. Relevant transform for the electric field strength gives $\hat{E}_{\xi, \eta, \zeta}^{\mathrm{e}}=\int_{-\infty}^{+\infty} d k_{\eta} \exp \left(i k_{\eta} \eta+i k \beta^{-1} \zeta-\kappa_{\xi} \xi\right) \hat{E}_{\xi, \eta, \zeta}^{\mathrm{e}}\left(k_{\eta}\right)$, where $\quad \widehat{E}_{\xi}^{\mathrm{e}}=\kappa_{\xi} \widehat{\varphi}^{\mathrm{e}}, \quad \widehat{E}_{\eta}^{\mathrm{e}}=-i k_{\eta} \widehat{\varphi}^{\mathrm{e}}$, $\widehat{E}_{\zeta}^{\mathrm{e}}=-i k \beta^{-1}\left(1-\beta^{2}\right) \hat{\varphi}^{\mathrm{e}} . \quad$ Replacing $\quad k_{\eta} \quad$ with $k_{z}=k_{\eta} \sin \theta_{\mathrm{e}}+k \beta^{-1} \cos \theta_{\mathrm{e}}$ and taking

$$
k_{x}^{\prime}=\left(\sin \theta_{\mathrm{e}}\right)^{-1}\left(k \beta^{-1}-k_{z} \cos \theta_{\mathrm{e}}\right),
$$

one gets $\hat{F}_{z}^{\mathrm{e}}=\int_{-\infty}^{+\infty} d k_{z} \exp \left(i k_{z} z+i k_{x}^{\prime} x^{\prime}\right) \tilde{F}_{z}^{\mathrm{e}}\left(k_{z}, \xi\right)$, where

$$
\begin{gathered}
\tilde{E}_{z}^{\mathrm{e}}\left(k_{z}, \xi\right)=i\left(\sin \theta_{\mathrm{e}}\right)^{-1}\left(k \beta \cos \theta_{\mathrm{e}}-k_{z}\right) \hat{\varphi}^{\mathrm{e}} \exp \left(-\kappa_{\xi} \xi\right), \\
\tilde{H}_{z}^{\mathrm{e}}\left(k_{z}, \xi\right)=\beta \kappa_{\xi} \widehat{\varphi}^{\mathrm{e}} \exp \left(-\kappa_{\xi} \xi\right) .
\end{gathered}
$$

That is, for the given $k_{z}$, the wedge is illuminated by one E-wave and one H-wave. They decrease exponentially in the direction $y^{\prime}$. Their amplitudes, $\tilde{E}_{z}^{\mathrm{e}}\left(k_{z}, \xi_{\mathrm{e}}\right)$ and $\tilde{H}_{z}^{\mathrm{e}}\left(k_{z}, \xi_{\mathrm{e}}\right)$, depend on $\xi_{\mathrm{e}}$ through the factor $\exp \left(-\kappa_{\xi} \xi_{\mathrm{e}}\right), \quad$ where $\quad \kappa_{\xi}=\left(f_{\perp}^{2}+k_{\eta}^{2}\right)^{1 / 2}$, $k_{\eta}=\left(\beta \sin \theta_{\mathrm{e}}\right)^{-1}\left(\beta k_{z}-k \cos \theta_{\mathrm{e}}\right)$.

The longitudinal field strength components for the sum of the incident and scattered waves are given by 2D distributions $F_{z}\left(k_{z}\right)=\tilde{F}_{z}^{\mathrm{e}}\left(k_{z}, \xi_{\mathrm{e}}\right) f_{z}\left(\chi_{F}, \rho, \varphi\right)$, where the quantities $\chi_{E}, \chi_{H}$, and $w_{\mathrm{i}}$ are dependent on the ratio $c_{z}=k_{z} / k$ (through (1), (3), and (4)). The 3D distributions for the set of waves are given by the equality $\hat{F}_{z}(r, \theta, \varphi)=\int_{-\infty}^{+\infty} d k_{z} \exp \left(i k_{z} z\right) F_{z}\left(k_{z}\right)$.

In the wave zone of $2 \mathrm{D}$ space, where $k_{\perp} \rho>>1$, the angular distribution of amplitude is obtained in [5], by the stationary phase method,

$$
f_{z}\left(\chi_{F}, \rho, \varphi\right) \approx\left[i /\left(2 \pi k_{\perp} \rho\right)\right]^{1 / 2} \exp \left(i k_{\perp} \rho\right) \Delta_{F}\left(c_{z}, \varphi\right),
$$

where $\Delta_{F}\left(c_{z}, \varphi\right)=\sum_{\{ \pm\}}\left[ \pm s_{z}\left(\chi_{F}, \varphi \mp \pi\right)\right]$. The incident and reflected waves related to the uniform charge motion are exponentially decreased in relevant direction, and they do not contribute to the radiation field. Similarly, in the wave zone of 3D space, where $k r>>1$, one gets

$$
\hat{F}_{z}(r, \theta, \varphi) \approx \exp (i k r) r^{-1} \tilde{F}_{z}^{\mathrm{e}}\left(k \cos \theta, \xi_{\mathrm{e}}\right) \Delta_{F}(\cos \theta, \varphi) .
$$

For the components of E- and $\mathrm{H}$ - waves, respectively, there are the relations $-E_{z} H_{\varphi}^{*} \approx\left|E_{z}\right|^{2} k / k_{\perp}$ and $E_{\varphi} H_{z}^{*} \approx\left|H_{z}\right|^{2} k / k_{\perp}$, with asterisk denoting the complex conjugate. The substitution $k_{z}=k \cos \theta$, corresponding to the arguments of the functions in (5), implies $c_{z}=\cos \theta$,

$$
w_{\mathrm{i}}=\varphi_{\mathrm{e}}-\pi+i \Lambda(\theta), \kappa_{\xi}=k\left(\beta \sin \theta_{\mathrm{e}}\right)^{-1} D^{1 / 2}(\theta),
$$

where

$\Lambda(\theta)=\operatorname{arcosh}\left[\left(1-\beta \cos \theta \cos \theta_{\mathrm{e}}\right) /\left(\beta \sin \theta \sin \theta_{\mathrm{e}}\right)\right]$,

$D(\theta)=\left(1-\beta^{2}\right) \sin ^{2} \theta_{\mathrm{e}}+\left(\beta \cos \theta-\cos \theta_{\mathrm{e}}\right)^{2}$.

The radiation field distribution depends on $\xi_{\mathrm{e}}$ through the factor $\exp \left[-k \xi_{\mathrm{e}}\left(\beta \sin \theta_{\mathrm{e}}\right)^{-1} D^{1 / 2}(\theta)\right]$, which is independent on $\varphi$. As a result, if the particle motion line is translated parallel to itself then relative distribution of the radiation field with respect to azimuth 
angle $\varphi$, at the given frequency, for the given polar angle $\theta$, is not changed, the power flux density for the different $\varphi$ is changed in accordance with the same factor written just above. Such azimuthal invariance of the radiation field takes place for the arbitrary $z$ uniform structure, but under the condition of the plane existence, with respect to which the structure and the particle motion line are in the different half-spaces. In such conditions the scattered field distribution is fully determined by the 'incident' field at the mentioned plane, and the Fourier transforms of the 'incident' field components depend on $\xi_{\mathrm{e}}$ exponentially, with increment dependent on $k_{z}$.

Proceeding from the equality

$$
W_{t}=(4 \pi)^{-1} c \int_{-\Phi}^{+\Phi} d \varphi \rho \int_{-\infty}^{+\infty} d z\left(\left[\vec{E}_{t} \vec{H}_{t}\right] \vec{e}_{\rho}\right),
$$

for the power flux through the cylinder with radius $\rho$, integrating it by time, moving from the functions to their Fourier transforms, and performing the limit transition $\rho \rightarrow \infty$, for the total radiated energy $W$ one gets

$$
W=4 \pi c \int_{0}^{\infty} d k \tilde{W},
$$

where $\tilde{W}=(4 \pi)^{-1} c k \int_{-k}^{+k} d k_{z} k_{\perp}^{-2} \sum_{F=E, H}\left|F_{z}\left(k_{z}\right)\right|^{2} I_{F}\left(c_{z}\right)$,

$$
I_{F}\left(c_{z}\right)=\int_{-\Phi}^{+\Phi} d \varphi\left|\Delta_{F}\left(c_{z}, \varphi\right)\right|^{2} .
$$

Replacing of integration variable, $k_{z}=k \cos \vartheta$, gives

$$
\begin{gathered}
\tilde{W}=\left(16 \pi^{3} c\right)^{-1} e_{0}^{2} \int_{0}^{\pi} d \vartheta(\sin \vartheta)^{-1} \exp \left(-2 \kappa_{\xi} \xi_{\mathrm{e}}\right) \times \\
\times\left[I_{H}(\cos \vartheta)+I_{E}(\cos \vartheta)\left(\cos \vartheta-\beta \cos \theta_{\mathrm{e}}\right)^{2} / D(\vartheta)\right] .
\end{gathered}
$$

\section{RESULTS}

In the case of perfect conductivity, $\operatorname{Im} \chi_{E}=+\infty$, $\chi_{H}=0$, one gets $s_{z}\left(\chi_{E}, \varphi\right)=s_{0}(\varphi), s_{z}\left(\chi_{H}, \varphi\right)=s_{1}(\varphi)$, where $s_{1}(\varphi)=k_{\Phi} \cos \left(k_{\Phi} \varphi\right)\left[\sin \left(k_{\Phi} \varphi\right)-\sin \left(k_{\Phi} w_{\mathrm{i}}\right)\right]^{-1}$, and integration in (7) gives

$$
\begin{gathered}
I_{E, H}(\cos \vartheta)=2 \pi k_{\Phi} \operatorname{coth}\left[k_{\Phi} \Lambda(\vartheta)\right] \times \\
\times \sin ^{2}\left(\pi k_{\Phi}\right) /\left\{\cosh ^{2}\left[k_{\Phi} \Lambda(\vartheta)\right]-\cos ^{2}\left(\pi k_{\Phi}\right)\right\} .
\end{gathered}
$$

As it follows from (6), (8), and (9), in the case of perfect conductivity, the total radiated energy does not depend on the angle $\varphi_{\mathrm{e}}$. So, if the particle motion line and the edge of wedge are fixed and the wedge is rotated around the edge then the total radiated energy is not changed.

After the next limit transition, to the perfectly conductive half-plane, for which $\Phi=\pi$, one gets

$$
\begin{gathered}
I_{E, H}(\cos \vartheta)=2 \pi \beta \sin \vartheta \sin \theta_{\mathrm{e}} / D^{1 / 2}(\vartheta), \\
W=3 e_{0}^{2} \gamma \beta^{2} \sin ^{2} \theta_{\mathrm{e}} /\left[8 \xi_{\mathrm{e}}\left(1-\beta^{2} \cos ^{2} \theta_{\mathrm{e}}\right)\right],
\end{gathered}
$$

in agreement with [2] and [3].

In the Fig. 2, an example of directional radiation pattern (the quantity $\left.c \exp \left(2 \kappa_{\xi} \xi_{\mathrm{e}}\right) d W /\left(e_{0}^{2} d \omega \sin \theta d \theta d \varphi\right)\right)$ is presented for the case $\beta=0.9, \alpha_{\mathrm{s}}=45^{\circ}, \Phi=150^{\circ}, \varphi_{\mathrm{e}}=45^{\circ}, \theta_{\mathrm{e}}=60^{\circ}$, $\theta=30^{\circ}$. Sloped straight lines correspond to the angles $\pm \Phi$ and $\varphi_{\mathrm{e}}$. The curves correspond to the frequencies, at which $|Z|=0,0.03,0.3$ (from right to left, at the horizontal wedge symmetry plane).
Appearance of nonzero impedance $\mathrm{Z}$ with $\alpha_{\mathrm{s}} \in(0, \pi / 2)$, may lead both to decrease of the total radiated energy and to its increase. The radiation field is determined by the charge and current distributions on the wedge faces. The increase of impedance corresponds to the resistance increase and may lead to decrease of the currents, and so, to the radiated power decrease. In particular, very small values of the radiation flux in the directions near to the symmetry plane in the Fig. 2, for the sufficiently large value of impedance, are connected with very small currents over the edge of wedge. On the other hand, near the surface with nonzero impedance, the surface wave exists. At $|Z|<<1$, its speed is near to the speed of light. When a particle moves to the edge almost parallel to a face of wedge then it effectively generates the waves, which speed projection on the particle motion direction is near to the particle speed. At the edge of wedge, a part of wave field transforms into a radiation field. In some conditions, an appearance and increase of impedance can yield an increase of the radiated energy.

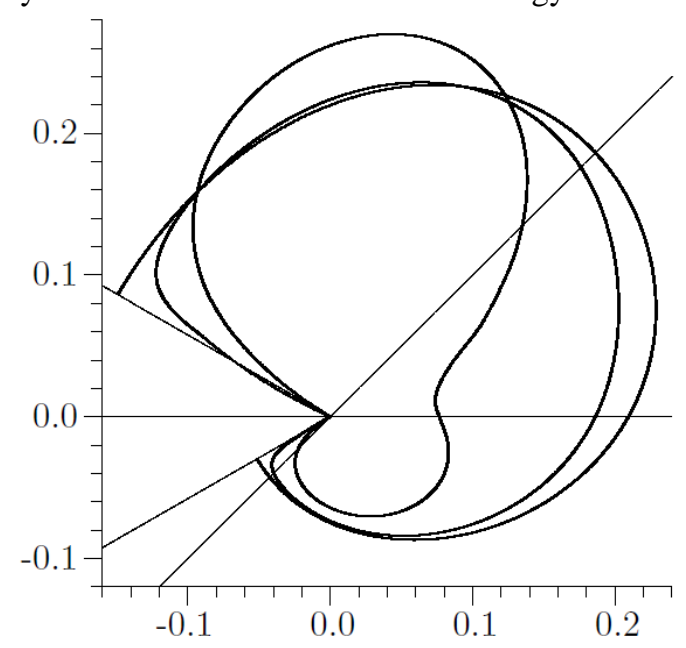

Fig. 2. Directional radiation pattern

To obtain simple approximate relations, it is expedient to consider the case when particle is relativistic, $\gamma>>1$, the distance between the motion line and edge is so large, that $\xi_{\mathrm{e}} \gg \gamma \delta_{\mathrm{s}}$, the value of $\sin \theta_{\mathrm{e}}$ is not small, and the motion line is almost parallel to the lower wedge face, so that the angle $\varphi_{\mathrm{f}}=\varphi_{\mathrm{e}}-\pi+\Phi$ is small, $\varphi_{\mathrm{f}}<<1$. Then the main part of energy is radiated within the angle $1 / \gamma$ near the motion directions of the particle and its mirror reflection in the lower wedge face, and for $\left|\theta-\theta_{\mathrm{e}}\right|<<1$ one gets the relations $\Lambda(\theta) \approx\left(1+\tau^{2}\right)^{1 / 2} /\left(\gamma \sin \theta_{\mathrm{e}}\right)$, $D(\theta) \approx \sin ^{2} \theta_{\mathrm{e}}\left(1+\tau^{2}\right) / \gamma^{2}, \kappa_{\xi} \approx k\left(1+\tau^{2}\right)^{1 / 2} / \gamma$, where $\tau=\gamma\left(\theta-\theta_{\mathrm{e}}\right)$. Due to the relation $\xi_{\mathrm{e}}>\gamma \delta_{\mathrm{s}}$ the factor $\exp \left(-2 \kappa_{\xi} \xi_{\mathrm{e}}\right)$ in (8) may be not small only at the frequencies, which obey the relation $k \delta_{\mathrm{s}}<<1$, and so, give $|Z|<<1$. That is, the relation $\xi_{\mathrm{e}}>>\gamma \delta_{\mathrm{s}}$ implies that 
the main contribution to the integral by $k$ in (6) is given by the frequencies, at which impedance is small. Using of the equality $\prod_{\{ \pm\}} \psi_{\Phi}((\pi / 2) \pm w)=\psi_{\Phi}^{2}(\pi / 2) \cos \left(k_{\Phi} w / 2\right)$

$$
\begin{aligned}
& \text { (from }[5]), \quad \text { for }\left|\theta-\theta_{\mathrm{e}}\right|<<1, \quad \text { gives } \\
& I_{E}(\cos \theta) \approx 2 \pi / \Lambda(\theta), \\
& I_{H}(\cos \theta) / I_{E}(\cos \theta) \approx 1-2\left[\Lambda(\theta) \operatorname{Im} \chi_{H}+\varphi_{\mathrm{f}} \operatorname{Re} \chi_{H}\right] \times \\
& \times\left\{\left(\varphi_{\mathrm{f}}+\operatorname{Re} \chi_{H}\right)^{2}+\left[\Lambda(\theta)+\operatorname{Im} \chi_{H}\right]^{2}\right\}^{-1} .
\end{aligned}
$$

Denoting $\tau_{\mathrm{f}}=\varphi_{\mathrm{f}} \gamma \sin \theta_{\mathrm{e}}, \tau_{\mathrm{Z}}=|Z| \gamma \sin ^{2} \theta_{\mathrm{e}}$, using the variable $\tau=\gamma\left(\theta-\theta_{\mathrm{e}}\right)$, and assuming that $\tau_{\mathrm{f}}$ and $\tau_{\mathrm{z}}$ are not very large, from $(8)$ one gets

$$
\begin{gathered}
\tilde{W}-\tilde{W}_{0} \approx\left(4 \pi^{2} c\right)^{-1} e_{0}^{2} \tau_{Z} \times \\
\times \int_{-\infty}^{+\infty} d \tau \exp \left[-2 k \xi_{\mathrm{e}}\left(1+\tau^{2}\right)^{1 / 2} / \gamma\right] \times \\
\times \cos \alpha_{\mathrm{s}}\left[\tan \alpha_{\mathrm{s}}-\tau_{\mathrm{f}} /\left(1+\tau^{2}\right)^{1 / 2}\right] / G(\tau),
\end{gathered}
$$

where $G(\tau)=\left[\left(1+\tau^{2}\right)^{1 / 2}-\tau_{\mathrm{Z}} \sin \alpha_{\mathrm{s}}\right]^{2}+\left(\tau_{\mathrm{f}}+\tau_{\mathrm{Z}} \cos \alpha_{\mathrm{s}}\right)^{2}$ and $\tilde{W}_{0}$ is the value of $\tilde{W}$ in the perfect conductivity case. From (6) and (10), changing the integration order, by $\tau$ and $k$, and changing the variable, $\tau=\cot \phi$, one gets

$$
W-W_{0} \approx\left(\pi \delta_{\mathrm{s}}\right)^{-1} e_{0}^{2}\left[\left(\gamma \delta_{\mathrm{s}}\right) /\left(2 \xi_{\mathrm{e}}\right)\right]^{p_{\mathrm{s}}+1} \gamma \sin ^{2} \theta_{\mathrm{e}} \times
$$

$\times \Gamma\left(p_{\mathrm{s}}+1\right) \cos \alpha_{\mathrm{s}} S\left(p_{\mathrm{s}}+1, \tau_{\mathrm{f}}^{2}\right)\left[\tan \alpha_{\mathrm{s}}-R\left(p_{\mathrm{s}}+1, \tau_{\mathrm{f}}\right)\right],(11)$

where $W_{0}$ is related to the in the perfect conductivity $\begin{array}{lll}\text { case }, \quad \Gamma \quad \text { is } & \text { gamma-function, } \\ R(p, \tau)=\tau S\left(p+1, \tau^{2}\right) / S\left(p, \tau^{2}\right), & \end{array}$

$$
S(p, q)=\int_{0}^{\pi} d \phi(\sin \phi)^{p} /\left(1+q \sin ^{2} \phi\right) .
$$

If the faces are resistive $\left(\delta_{\mathrm{s}}>0, \cos \alpha_{\mathrm{s}}>0\right)$ then the right hand side of (11) may be both positive and negative. In the case of normal skin effect it is zero at $\tau_{\mathrm{f}} \approx 1.194$. For smaller angle $\varphi_{\mathrm{f}}$ it is positive and appearance of resistance leads to increase of the radiated energy.

\section{CONCLUSIONS}

The wide-band electromagnetic pulse may be generated by particle bunch created in the pulse accelerator and passing by antenna. If the bunch is relativistic then the main part of radiation is emitted in the directions near to the direction of the bunch motion. The case of plasma wedge-form antenna and single charge moving uniformly is considered in impedance approximation. For the perfectly conductive wedge (zero impedance) it is shown that the total radiated energy does not depend on the direction of the charge motion line projection on a plane perpendicular to the edge of wedge. That is, if the particle motion line and the edge are fixed and the wedge is rotated around the edge then the total radiated energy is not changed. Appearance and increase of impedance may lead both to decrease and to increase of the total radiated energy. Its decrease can be caused by the decrease of the surface currents, through the resistance increase. The increase of the total radiated energy is observed in the conditions, favorable for the surface wave generation, when the charge moves to the edge almost parallel to a face of wedge and the particle velocity is near to the wave velocity.

\section{REFERENCES}

1. V.A. Balakirev, N.I. Gaponenko, A.M. Gorban', et al. Excitement of TEM-horn antenna by impulsive relativistic electron beam // Problems of Atomic Science and Technology. Series «Plasma Physics» (5). 2000, № 3, p. 118-119.

2. A.P. Kazantsev, G.I. Surdutovich. The radiation of the charged particle flying near the conductive screen // Dokl. Akad. Nauk SSSR. 1962, v. 147, № 1, p. $74-77$ (in Russian).

3. A.P. Potylitsyn, M.I. Ryazanov, M.N. Strikhanov, A.A. Tishchenko. Diffraction radiation of the charged particles. Tomsk, 2008, $347 \mathrm{p}$.

4. G.D. Malyuzhinets. The excitation, reflection and radiation of surface waves in a wedge-like region with given face impedances // Dokl. Akad. Nauk SSSR. 1958, v. 121, № 3, p. 436-439 (in Russian).

5. V.M. Babich, M.A. Lyalinov, V.E. Grikurov. Sommerfeld-Malyuzhinets technique in diffraction theory. Saint-Petersburg, 2004, 103 p.

Article received 08.01.2021

\section{ИЗЛУЧЕНИЕ ЗАРЯДА ПРИ ДВИЖЕНИИ МИМО ИМПЕДАНСНОГО КЛИНА}

\section{В. Остроуико}

Рассмотрено в импедансном приближении дифракционное излучение, созданное зарядом при движении мимо плазменного клина. В пределе идеальной проводимости полная излученная энергия не изменяется при повороте клина вокруг ребра, фиксированного вместе с линией движения заряда. Увеличение импеданса может вести к увеличению полной излученной энергии благодаря эффективному созданию поверхностной волны, когда движение заряда к ребру почти параллельно к грани клина.

\section{ВИПРОМІНЮВАННЯ ЗАРЯДУ ПРИ РУСІ ПОВЗ ІМПЕДАНСНОГО КЛИНА}

\section{В. Остроушко}

Розглянуте в імпедансному наближенні дифракційне випромінювання, утворене зарядом при русі повз плазмового клина. У граничному випадку ідеальної провідності загальна випромінена енергія не змінюється при обертанні клину навколо ребра, фіксованого разом з лінією руху заряду. Збільшення імпеданса може вести до збільшення загальної випроміненої енергії завдяки ефективному утворенню поверхневої хвилі, коли рух заряду до ребра майже паралельний до грані клину. 